\title{
PRÁCTICA BASADA EN EVIDENCIA EN EL ÁREA PEDIÁTRICA EN CHILE, UN DESAFÍO PENDIENTE.
}

\author{
EVIDENCE BASED PRACTICE IN PAEDIATRIC SETTINGS IN CHILE, A PENDING
} CHALLENGE.

\section{Jenniffer García Rojas'; Jodie Copley²; Merrill Turpin³; Natalia Peña Jeldes ${ }^{4}$}

\begin{abstract}
RESUMEN
La práctica basada en evidencia (PBE) promueve el uso de evidencia científica, experticia clínica, información acerca del cliente y del contexto de práctica. Su objetivo es mejorar estándares profesionales a través de la creación y uso de información de calidad en la toma de decisiones clínicas. Terapia Ocupacional ha sido desafiada a adoptar PBE globalmente, sin embargo, aún no es una realidad mundial. En Chile actualmente se gradúa un número creciente de terapeutas ocupacionales, constituyéndose en un interesante ejemplo para explorar qué recursos de información usan los terapeutas ocupacionales, de qué manera desarrollan conocimiento y lo integran en la práctica pediátrica.

Métodos: "Interpretive description" fue utilizado como marco metodológico en este estudio, e involucró como informantes a diez terapeutas ocupacionales quienes participaron en entrevistas en profundidad. Los datos fueron transcritos, traducidos al inglés, codificados y analizados temáticamente. Las interpretaciones preliminares fueron enviadas a los participantes, quienes retroalimentaron el análisis de los datos.

Resultados: Para tomar decisiones clínicas, Los participantes usaron primordialmente experticia profesional e intuición. El acceso a desarrollo profesional continuo, a evidencia científica e información acerca de práctica contemporánea parece limitado. Los programas de formación continua que se encuentran disponibles impactan significativamente la práctica pediátrica en Chile.

Discusión: El limitado acceso a la evidencia científica y restringida conexión con la comunidad científica global, en contextos de cultura de investigación emergente, puede influir en que los terapeutas ocupacionales tomen decisiones basados en información cuya calidad no ha sido evaluada por pares. Es relevante desarrollar marcos de razonamiento clínico basado en evidencia en Chile. El estatus de la profesión podría potenciarse empoderando al colegio profesional de Chile, respaldando programas de educación continua actualizados, promoviendo cultura de investigación, conexión con la comunidad científica global y mejorando estándares de práctica.
\end{abstract}

\section{PALABRAS CLAVE}

Práctica basada en evidencia, terapia ocupacional, toma de decisiones clínicas, pediatría, Chile.

1 Terapeuta Ocupacional y Licenciada en Ciencias de la Ocupación Humana, Universidad de Chile. MPhil, PhD student, The University of Queensland orcid.org/0000-0002-7482-9220/ ResearchID C-3284-2017. Correo postal: 1/22 Warren Street, St Lucia. Brisbane, QLD 4067. Australia. Correo electrónico: j.garcia@uq.net.au. Teléfono: +61406508055

2 Bachelor of Occupational Therapy, The University of Queensland PhD, The University of Queensland. Assistant Professor, School of health and rehabilitation Sciences, orcid.org/0000-0001-8373-5990. Correo postal: Therapies Building \#84, The University of Queensland, St Lucia QLD 4072, Australia. Correo electrónico: j.copley@uq.edu.au.

3 Bachelor of Occupational Therapy, The University of Queensland. GDipCouns, Brisbane College of Advanced Education. Master of Occupational Therapy. The University of Queensland. PhD, The University of Queensland. Senior lecturer, School of health and Rehabilitation Sciences, The University of Queensland. orcid.org/0000-0001-7946-0883Correo postal : Therapies Building \#84, The University of Queensland, St Lucia QLD 4072, Australia. Correo electrónico: m.turpin@uq.edu.au. Teléfono: +61 733656491.

4 Terapeuta Ocupacional y Licenciada en Ciencias de la Ocupación Humana, Universidad de Playa Ancha. Afiliación: Escuela de Salud, Universidad Viña del Mar. Correo electrónico: nataliapenajeldes@gmail.com. Teléfono: +56982145128. 


\section{ABSTRACT}

Evidence-based practice (EBP) fosters the use of research evidence, clinical expertise, information about the patient and the practice context. It aims to support high quality practice through providing and using sound evidence for decision-making. Occupational therapists have been challenged to adopt EBP as a global approach, nevertheless it is not yet a worldwide reality. Chile, currently graduating an increasing number of therapists, provides a useful case for exploring which sources of information are accessed by therapists and how knowledge is build and integrated in paediatric practice.

Methods: Using Interpretive description as the methodological framework, ten Chilean occupational therapists completed in-depth interviews. Data were transcribed verbatim and then translated into English, coded and analyzed thematically. Preliminary interpretations were returned to the participants that provided feedback, enriching data analysis.

Results: Participants predominantly rely on intuition and clinical expertise when making decisions. Access to continuing professional development, research knowledge and information about contemporary practice seemed to be limited. Professional training available strongly influences all aspects of their practice.

Discussion: Scarce access to research knowledge and lack of connection with the global scientific community, in the context of emergent research culture, can lead occupational therapists to rely on information that has not undergone quality evaluation. The development of a clinical reasoning framework based on evidence in Chile results relevant. The status of the profession may be enhanced through empowering the professional association, supporting high quality and updated professional training, promoting research culture, and enhancing the standards of practice in Chile.

\section{KEYWORDS}

evidence based practice, occupational therapy, clinical decision-making, paediatrics, Chile.

Recibido: 30/03/2017

Aceptado: 23/10/2017 


\section{INTRODUCCIÓN}

La práctica basada en evidencia (PBE) es trascendental para profesionales de la salud, debido al crecimiento exponencial del conocimiento a nivel mundial, y a la necesidad de probar la efectividad de los servicios de salud ofrecidos (Lin et al., 2010; Thomas et al., 2012). El concepto original, medicina basada en la evidencia, fue concebido en los años 70' en Canadá por la Escuela de Medicina de McMaster University, que decidió crear un nuevo programa en que dicha carrera duraba sólo 3 años. La estrategia utilizada fue enseñar a los alumnos cómo aprender efectivamente por sí mismos, en lugar de entregarles todos los conocimientos que debían adquirir (Hoffmann et al., 2013). Este hito marcó el origen de la práctica basada en evidencia, y desde entonces, ha ganado relevancia en forma creciente.

PBE tiene un rol crucial en el área de salud, permitiendo a los profesionales tomar mejores decisiones clínicas, y optimizando los resultados obtenidos por sus clientes (Hoffman et al., 2013) En rehabilitación, PBE se ha convertido en un "paradigma fundamental" en la práctica clínica (Tse et al., 2004, p.269). En el año 2000, un modelo específico para terapeutas ocupacionales fue propuesto (Bennett \& Bennett, 2000), el cual ha sido actualizado y redefinido, incorporando "el uso de razonamiento clínico para integrar información proveniente de 4 fuentes de información en la toma de decisiones clínicas: evidencia científica, experticia clínica, los valores, preferencias y circunstancias del paciente, e información del contexto de práctica" (Hoffmann et al., 2013, p 4).

Para lograr PBE, los terapeutas ocupacionales necesitan acceder a información científica, considerada como un recurso de información clave (Rycroft-Malone, 2004). Sin embargo, el uso e integración de evidencia científica en la práctica diaria ha sido reconocida como una tarea desafiante (Colquhoun et al., 2010), por lo cual los terapeutas ocupacionales necesitan soporte. Thomas \& Law (2013) señalaron que la falta de conocimientos para evaluar e interpretar hallazgos científicos, o falta de evidencia científica que sea relevante para la clínica, pueden constituir barreras para la PBE. Adicionalmente, las autoras han identificado factores facilitadores, tales como realizar investigación, establecer equipos de investigación entre clínicos y docentes de Universidades, y realización de estudios de postgrado. Paralelamente se han realizado acciones concretas, como la creación de OTseeker, base de datos específica de Terapia Ocupacional y de libre

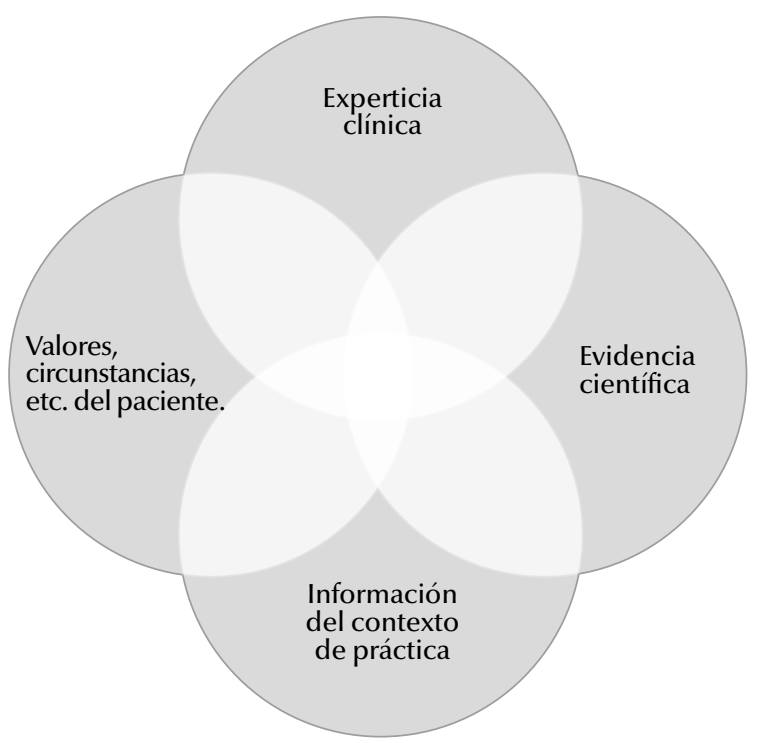

FIGURA 1: Práctica basada en evidencia consiste en el uso del razonamiento clinico para integrar 4 recursos de información: evidencia cientifica, experticia clínica, los valores, circunstancias y preferencias del paciente, e información del contexto de práctica. Figura y texto extraídos y traducidos de Evidence based practice across health professions. Hoffmann, Bennett \& Del Mar, 2013, p.4)

acceso (Bennett et al., 2003). Se ha enfatizado además, la relevancia de usar conocimientos y técnicas que sean coherentes con la filosofía de la profesión y su concepto central de ocupación (Gustafsson et al., 2014).

Experticia clínica es el segundo tipo de información requerida para la PBE, definida como la "competencia y el juicio" clínico que profesionales de salud desarrollan en base a su práctica profesional (Sackett et al., 1996, p.71). Se ha descrito que los terapeutas ocupacionales fundamentan frecuentemente sus decisiones en base a su experticia clínica (Bennett et al., 2003). Sin embargo, debido a que el tipo de conocimiento que proviene de la experticia clínica es intuitivo y tácito, ha sido devaluado en contextos científicos, particularmente si es el único criterio usado para tomar decisiones (Rycroft-Malone et al., 2004). Para desarrollar experticia, los terapeutas ocupacionales necesitan desarrollar un cuerpo de conocimientos que se nutra de estudios de postgrado (Alsop, 2013), y del contacto con redes profesionales, ya que a través de reuniones clínicas, supervisión sistemática de pares, y contar con un terapeuta ocupacional experto como mentor, es posible lograr excelencia en la práctica (Copley et al., 2010b). Países desarrollados han creado 
mecanismos adicionales para respaldar prácticas de calidad en terapia ocupacional. Por ejemplo, colegios profesionales e instituciones reguladoras tienen un rol fundamental en organización de actividades de formación continua, desarrollo de estándares para registro formal de profesionales, además de supervisar la calidad de la práctica (García et al., 2016).

Como tercer componente, las experiencias, valores y preferencias del paciente han sido considerados esenciales para la PBE (Rycroft-Malone et al., 2004), e identificados en terapia ocupacional como fundamentales desde los inicios de la profesión, los cuales continúan siendo eje central de modelos contemporáneos tales como la práctica centrada en el cliente (Mroz et al., 2015). Un estudio exploró qué tipo información usó una terapeuta ocupacional pediátrica experta para tomar decisiones clínicas, identificando que los resultados de evaluaciones formales, observaciones y entrevistas, era consideradas como lo más relevante. Esta información era frecuentemente actualizada a través de discusiones con el niño, su familia y personas significativas de su entorno (Copley et al., 2010a), hallazgos que son coherentes con el concepto central de la PBE, donde el paciente y su familia y/o entorno juegan un rol fundamental.

El contexto de la práctica clínica constituye el cuarto recurso de información de la PBE. En terapia ocupacional se han identificado múltiples variables contextuales que influyen sobre el razonamiento clínico y toma de decisiones (Shaafarodi et al., 2014). Por ejemplo, algunos estudios han señalado que factores como los recursos, el tiempo y mobiliario disponible determinaron el tratamiento escogido para niños con trastornos de aprendizaje (Copley et al., 2008). Adicionalmente, se ha identificado que la presencia de líderes que promuevan el uso de evidencia científica en el contexto de trabajo (Thomas \& Law, 2013), libre acceso a bases de datos, tiempo disponible durante la jornada laboral (Humphris, 2000) y apropiada carga laboral (Dysart \& Tomlin, 2002) facilitan la PBE.

La Federación Mundial de Terapeutas Ocupacionales ha desplegado parte de su agenda fomentando la PBE (Piergrossi, 2004), y la comunidad profesional ha sido llamada a desarrollar un "plan estratégico a nivel mundial en torno a la Terapia Ocupacional Basada en Evidencia (тове) ...promoviendo el debate y la acción colaborativa, especialmente para aquellos que viven en países en vías de desarrollo" (Ilott et al., 2006). En décadas recientes, la PBE ha capturado la atención de las revistas científicas disciplinares más relevantes a nivel mundial, primordialmente publicadas por países desarrollados y angloparlantes (Bannigan, 2011; Buchanan, 2011). Sin embargo, temas como la PBE e investigación en muchos países y regiones en vías de desarrollo tales como "Chile y Latinoaméricana, este ha sido un tema escasamente explorado" (Aravena et al., 2016, p 176). Debido a estas razones, este proyecto de investigación fue conducido en Chile entre los años 2015 y 2016, y enfocado en el campo de pediatría, el cual es reconocido como una de las áreas tempranamente desarrolladas y más comunes de la práctica en Chile (Gómez, 2012).

Este proyecto fue ejecutado en torno a dos preguntas de investigación: ¿qué recursos de información se encuentran disponibles y son usados por terapeutas ocupacionales pediátricos en Chile para tomar decisiones clínicas?, y ¿ de qué manera, los terapeutas ocupacionales chilenos que trabajan en pediatría, construyen y fundamentan sus conocimientos disciplinares?

\section{MÉTOdo}

Este estudio fue desarrollado en el marco de un programa de Master de investigación en la Universidad de Queensland (UQ), Australia. Previo al inicio de recolección de datos, el departamento de ética en investigación con seres humanos de UQ otorgó aprobación a este proyecto. Debido a que las entrevistas fueron realizadas fuera el horario de trabajo de los participantes, adicionalmente y en cumplimiento con las normativas éticas internacionales, se solicitó aprobación ética al Colegio de Terapeutas Ocupacionales de Chile en su calidad de Gatekeeper, ante lo cual no se recibió respuesta. Los participantes recibieron un documento informativo explicando los objetivos del proyecto y a la potencial publicación de resultados de éste, y entregaron su consentimiento informado previo al inicio de la investigación.

El marco metodológico elegido fue "Interpretive description" (Thorne, 2008), considerada como la metodología de investigación cualitativa más apropiada, ya que fue creada para contextos clínicos en el área de salud. Se seleccionó el método a conveniencia para reclutar"informantes claves" (Thorne, 2008, p.91). Adicionalmente, se consideraron aspectos culturales de Chile tales como el uso extendido del "compadrazgo" (Bazoret, 2006, p.2); así, la investigadora principal usó su red de contactos profesionales para reclutar a los participantes, y a través 
de ellos invitar a otros terapeutas ocupacionales usando la estrategia llamada bola de nieve. Esta técnica fue necesaria debido a que inicialmente se solicitó al Colegio de Terapeutas Ocupacionales de Chile distribuir una invitación entre sus asociados, pero el equipo investigador no recibió respuestas de algún interesado en participar.

Dos hombres y ocho mujeres fueron reclutados, asegurando la mayor variedad posible en sus perfiles profesionales: Terapeutas Ocupacionales con 5 meses a 30 años de experiencia en pediatría, con título profesional, Licenciatura, Diplomas o Magíster. Se reclutaron participantes de la zona Norte, Centro y Sur de Chile, que trabajaran en centros públicos, privados u organizaciones no gubernamentales (ONG), con diversidad en el tipo de pacientes pediátricos atendidos. Los nombres de los participantes fueron reemplazados por pseudónimos para resguardar confidencialidad.

Una entrevista en profundidad, semi-estructurada, fue desarrollada por el equipo investigador. Dos miembros del equipo (terapeutas ocupacionales chilenas, una ubicada en Australia y otra en Chile, ambas con experiencia clínica en pediatría) recibieron entrenamiento para conducir entrevistas. Se realizó una fase piloto entrevistando tres terapeutas ocupacionales pediátricos en Chile, previo a la recolección de datos, para asegurar la pertinencia cultural. Los participantes completaron el perfil demográfico, y las entrevistas fueron realizadas en persona, o a través de videoconferencias en el caso de terapeutas ocupacionales de regiones, y grabadas. Los audios fueron transcritos literalmente, traducidos al inglés y revisados por un traductor profesional. Tres miembros del equipo investigador desarrollaron una estrategia de codificación, y los datos fueron ordenados inductivamente, creando una matriz de categorías y códigos, usando software NVivo 11 para el manejo de datos. Para incrementar la credibilidad del análisis, interpretaciones preliminares del equipo investigador fueron enviadas a los participantes para su revisión crítica (Thorne, 2008). Ocho de los 10 participantes del proyecto entregaron feedback, enriqueciendo el análisis temático desarrollado por el equipo investigador.

\section{Resultados}

Los temas emergentes del análisis de datos, revelaron qué factores influyen en la toma de decisiones clínicas, y de qué manera los participantes desarrollan su conocimiento disciplinar.

\section{INFORMACIÓN UTILIZADA PARA LA TOMA DE DECISIONES EN PEDIATRÍA}

Para la toma de decisiones, los participantes de este proyecto consideraron principalmente la información recogida acerca del paciente/cliente, y su experticia clínica (ver FIGURA 2).

\section{FIGURA 2}

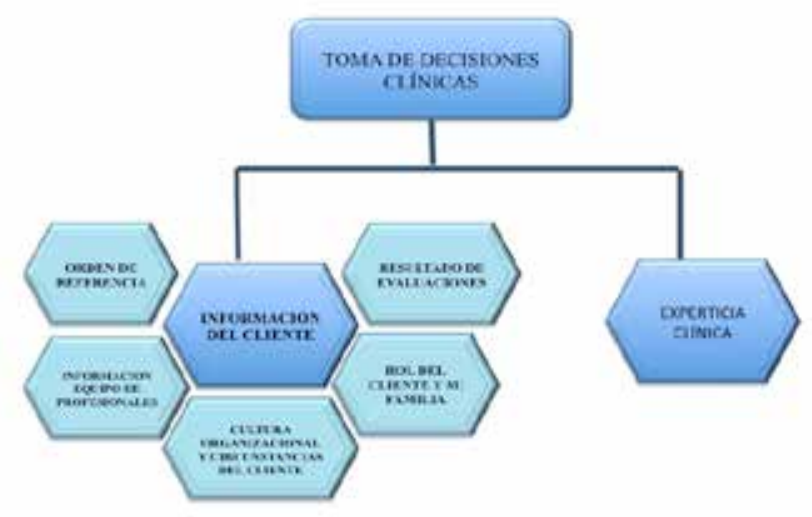

Tabla 2: Fuentes de información usadas por terapeutas ocupacionales para la toma de decisiones clínicas. Elaborado por equipo investigador.

A. Información acerca del paciente, con cinco factores descritos como influyentes:

- Orden de referencia/Hoja de derivación: considerada por siete participantes al momento de tomar decisiones. Éstas solicitaban a los terapeutas ocupacionales trabajar en objetivos determinados, aplicar evaluaciones específicas o diagnosticar al niño. Dos participantes mencionaron dificultades, debido a que algunos pacientes fueron diagnosticados intencionalmente en forma errónea con Trastorno de déficit Atencional e Hiperactividad (TDAH). El propósito era asegurar que los pacientes fueran aceptados en el centro, ya que este diagnóstico constituía un criterio de ingreso. 
- Resultados de la evaluación: Considerado como elemento clave para la toma de decisiones. Las estrategias usadas para evaluar a los niños incluían observación clínica, y en menor medida, aplicación de evaluaciones estandarizadas. Las actividades de la vida diaria, grafomotricidad, coordinación motriz, comportamiento, postura y control motor en pacientes con condiciones neurológicas, fueron evaluadas primordialmente a través de la observación. Siete participantes señalaron que la observación es comúnmente usada para evaluar, y la única estrategia usada por una participante con 10 años de experiencia:

"Para evaluar niños, sólo uso mi observación clínica" (JACQUIE).

Los participantes que trabajan en centros privados tuvieron acceso a un mayor número y variedad de herramientas de evaluación que terapeutas ocupacionales en centros públicos.

- Información entregada por miembros del equipo: Considerada como relevante por la mayoría de los participantes. Los terapeutas ocupacionales que trabajaban en hospitales señalaron que el equipo médico es trascendental, y que obtenían información de ellos antes de evaluar al paciente. Adicionalmente, un participante con menos de 6 meses de experiencia trabajando en una Escuela especial señaló que definía los objetivos de tratamiento y las intervenciones basada en la opinión de la profesora del paciente.

- Rol del cliente y su familia: Las respuestas sugieren que la participación de la familia en el proceso de evaluación e intervención se limita a entregar información a los terapeutas ocupacionales acerca de las principales dificultades de los niños en sus casas y en el colegio, y completar cuestionarios. La mayoría de los padres no son autorizados a ser parte de las sesiones de tratamiento. En relación a los pacientes, los datos revelaron que los niños juegan un rol pasivo en la toma de decisiones, y la mayoría de los terapeutas establecía una relación vertical con sus clientes. Cuando los participantes fueron invitados a reflexionar acerca del rol de los niños, éstos señalaron que su opinión sí era considerada, al preguntarles acerca su percepción de las propias dificultades, tal como ejemplifica una participante con vasta experiencia clínica: "para considerar la opinión del niño?...Sí, hacemos esa pregunta ¿porqué crees que tus papás te trajeron aqui? (ROSARIO)

- Impacto de la cultura organizacional y circunstancias del cliente en la prestación de servicios: El modelo médico aparece como dominante en la mayoría de los casos, influyendo en el funcionamiento de servicios, el rol jugado por los terapeutas ocupacionales, los niños y sus familias. En la mayoría de los centros, todas las decisiones debían ser tomadas por médicos, aun cuando fueran pertinentes a Terapia Ocupacional. Las clases sociales a las cuales pertenecían los pacientes, al igual que la práctica social del "compadrazgo", emergieron como factores que influyen en la manera en que los clientes acceden a servicios de Terapia Ocupacional. Participantes que trabajaban en zonas remotas, mencionaron dificultades debido a que la profesión no es una disciplina conocida por todos los miembros del equipo de salud, lo que provocó falta de asignación de recursos y menor derivación de clientes a las unidades terapéuticas.

\section{B. Experticia clínica guiando toma de decisiones en la práctica}

Experticia clínica fue el segundo factor influyente en las decisiones de los participantes. El uso de la experiencia profesional e intuición fueron frecuentemente mencionados, independiente de los años de experiencia o la formación de postgrado que tuvieran. La participante con mayor experiencia clínica explicó que decisiones intuitivas respaldaban poderosamente su trabajo en pediatría. De la misma forma, una participante con 5 meses de experiencia profesional señaló:

"Especificamente, nosotros notenemos ninguna pauta de evaluación establecida, eso no se trabaja así... como te digo, no hay ninguna pauta específica que yo diga voy a completar esta pauta, o algo cuantitativo que me diga que este niño tiene problemas y sí necesito intervenir o no, es en base a mi opinión... yo no soy de usar libros, yo aprendo mucho de la experiencia. De la experiencia también basada en teoría, pero lo fundamental es la experiencia neta"(SARA). 


\section{DE QUÉ MANERA SE CONSTRUYE EL CONOCIMIENTO DISCIPLINAR}

Para construir su cuerpo de conocimiento disciplinar, se identificaron cuatro fuentes principales:

- Educación formal: Cuatro participantes mencionaron estudios de pregrado como relevantes en la construcción del conocimiento en pediatría. La mayoría de los participantes señalaron que gran parte de sus aprendizajes ocurrió en sus prácticas profesionales, y en el ejercicio clínico una vez graduados. En relación a estudios de postgrado, algunos participantes mencionaron escasez de programas de Terapia Ocupacional en pediatría en Chile, y en lo concreto, sólo un participante tenía estudios de Magíster en ciencias aplicadas. Los diplomas fueron comúnmente mencionados, pero la mayoría de estos programas pertenecían y eran impartidos por disciplinas distintas a Terapia Ocupacional. Este tipo de estudios parece impactar significativamente la práctica clínica de los terapeutas, tal como señala una de las participantes:

\section{"Aquí, hay toda una mirada que nos ha abierto los ojos, porque uno está siempre muy centrado siempre en los déficits de los niños. Parentalidad positiva nos ha abierto los ojos en términos de cuáles son las habilidades de los niños, de la mamá en relación con sus hijos" (ROSARIO).}

- Impacto de formación continua en la práctica clínica: Los participantes indicaron que los cursos de desarrollo profesional continuo (DPC) comúnmente consisten en workshops, seminarios y cursos de corta duración, principalmente organizados por personas o instituciones privadas. Los eventos de DPC disponibles influyeron poderosamente sobre la práctica. Entrenamiento en Integración Sensorial (IS) fue mencionado por todos los participantes. Éstos indicaron usar IS, aun cuando no tuvieran los recursos físicos necesarios para ello, con pacientes con un amplio abanico de diagnósticos tales como Autismo, TDAH, Trastornos de apego y comportamiento, Parálisis Cerebral, Traumatismo encéfalo-craneano, Enfermedades congénitas, Cáncer, Discapacidades intelectuales y motoras, Síndrome de Down y Retraso del desarrollo motor entre otras. Por ejemplo, una participante con más de 15 años de experiencia señaló:
"Yo básicamente uso integración sensorial, y principios de neurodesarrollo. Aun cuando hay niños que no tienen problemas de base de integración sensorial, muchos niños que se ven beneficiados con este enfoque. Entonces ocupo integración sensorial pura. Para niños con problemas motores uso teorías de desarrollo motor e integración sensorial, para niños con problemas de relaciones, ocupo integración sensorial y Floortime, que también es una técnica basada en integración sensorial" (CRISTINA).

Cuando se preguntó a los participantes cómo podrían mejorar la calidad de su práctica, la mayoría expresó el deseo de tomar o repetir cursos de IS que habían realizado anteriormente. Los participantes señalaron que actualmente las instituciones pediátricas exigen tener formación en IS para poder obtener puestos de trabajo. Adicionalmente, participantes de diversos contextos clínicos señalaron que las expectativas de profesores, padres y profesionales de la salud, es que los Terapeutas Ocupacionales usen IS para tratar a sus pacientes.

Las ofertas de formación en el Modelo de Ocupación Humana ( $\mathrm{MOH})$ fue el segundo tipo de eventos de educación continua más mencionados. En consecuencia, las herramientas de evaluación y tratamiento pertenecientes al $\mathrm{MOH}$, fueron frecuentemente citados después de IS, y los participantes señalaron tener fácil acceso a literatura y materiales relacionados al modelo. El relato de una participante que trabajaba en un hospital público, ejemplifica cómo el conocimiento disponible en relación a marcos de referencia y modelos de intervención, influye significativamente en la clínica. La profesional explicó que en los servicios de salud a nivel nacional se estableció un protocolo de tratamiento para niños con cáncer, que incluye servicios de Terapia Ocupacional basados en el $\mathrm{MOH}$. Este protocolo obliga a los terapeutas a aplicar herramientas de evaluación y establecer objetivos de tratamiento en base a este modelo.

La Teoría de Neurodesarrollo (TND) Bobath fue mencionada en tercer lugar, señalando que Bobath es el Gold standard para tratar niños con condiciones neurológicas. Los participantes también mencionaron haber recibido entrenamiento en Parentalidad positiva, Floortime, Yoga, Estrategias cognitivo-conductuales, Drama-terapia, Theraplay, Terapia asistida por perros, Hipoterapia, Flores de Bach, Masaje Shantala y Landen, además de Therapy-taping. 
Cinco participantes fueron consultados acerca de su conocimiento en torno a algunos modelos disciplinares que son parte de la práctica pediátrica contemporánea en otros países, como por ejemplo el modelo de Orientación cognitiva al desempeño ocupacional, la Terapia restrictiva de movimiento, programa de entrenamiento bimanual, entrenamiento orientado a las metas, o el modelo de práctica centrada en la ocupación (Rodger, 2010). Sin embargo, sólo la Terapia restrictiva fue familiar para un participante, y el resto de los modelos fueron desconocidos para los participantes encuestados.

- Poder de las redes profesionales informales: Recurso ampliamente usado por los participantes, en particular de manera informal. Nueve participantes señalaron que sus contactos profesionales-personales les ayudan a resolver dudas, proveen consejos acerca de la práctica, y comparten información acerca de cursos de formación y compra de implementos. Los medios de comunicación fueron Facebook, Whatsapp e email. Seis participantes mencionaron a profesionales de otras disciplinas, de quienes obtenían conocimiento y destrezas profesionales. Terapeutas ocupacionales que trabajaban en áreas remotas señalaron que tienen acceso restringido o nulo a redes profesionales.

Ningún participante mencionó espontáneamente supervisión de pares o tener mentores para obtener conocimiento. Cuando se les preguntó al respecto, seis participantes señalaron no poder identificar a nadie como mentor. Sólo un Terapeuta ocupacional había tenido experiencias de supervisión en curso de especialización impartido por docentes estadounidenses.

- Barreras en el acceso al conocimiento: Los datos reflejaron que los participantes enfrentaban numerosas barreras para acceder al conocimiento. Mencionaron que la mayoría de las opciones de formación son ofrecidas en la ciudad de Santiago, con escasas ofertas para regiones, de elevado costo y con docentes primordialmente extranjeros. El acceso a entrenamiento en herramientas de evaluación fue identificado como limitado, varios participantes habían recibido entrenamiento por parte de colegas, o habían descargado pautas de evaluación de internet, algunos de ellos sin contar con manuales de aplicación. Algunos terapeutas ocupacionales reportaron el uso de versiones re- sumidas de evaluaciones originalmente creadas en países angloparlantes, pero desconocían el autor de dichas traducciones y resúmenes, así como su validez. La falta de apoyo por parte de jefaturas y empleadores para tomar cursos de perfeccionamiento fue mencionado por los participantes que trabajaban en el área pública, y en menor medida por algunos terapeutas del área privada.

Ocho participantes enfrentaron barreras en relación al uso de artículos científicos, tales como falta de acceso a bases de datos, falta de herramientas para comprender literatura científica y limitadas publicaciones en idiomas diferentes al inglés. Uno de los dos únicos participantes que tenía parcial acceso a literatura en forma autónoma, señaló que para obtener todos los artículos que necesita debía hacer uso de una página web y un grupo de Facebook que comparten artículos gratuitamente o los obtienen a través de la piratería. Tres participantes sólo accedían a artículos que les entregan los docentes de los cursos de postítulo a los cuales asisten. Adicionalmente, sólo los participantes con menos de 3 años de egreso habrían recibido entrenamiento en estrategias de búsqueda de artículos científicos, sin embargo nunca habían usado bases de datos debido a no tener acceso a ellas. Estos participantes relataron que en la universidad les pedían usar artículos científicos para justificar los planes de tratamiento, pero sólo en los exámenes finales. Fue comúnmente mencionado que la información profesional era obtenida a través de páginas de Facebook, blogs y otros sitios en internet.

\section{Discusión}

Dos hallazgos principales emergieron de este proyecto de investigación: La toma de decisiones clínicas fue influenciada por la experticia profesional de los terapeutas ocupacionales y la información recogida en relación al paciente, sin embargo, el uso de literatura científica no fue identificada como un elemento central para los participantes. Luego, el acceso al conocimiento presentó numerosas barreras, frente a lo cual los terapeutas ocupacionales confiaban en fuentes de información disponible, que no siempre habían sido sometidas a evaluación de pares.

La literatura sugiere que la evidencia científica no es regularmente incorporada en la práctica clínica (Hu 
et al., 2012), lo cual es consistente con los hallazgos de este estudio. Aun cuando los participantes buscaban activamente acceder al conocimiento disciplinar, el uso de la evidencia científica no parece ser considerado al momento de tomar decisiones. Países no-angloparlantes y en vías de desarrollo enfrentan obstáculos adicionales que han sido escasamente discutidos en la literatura, en términos de cómo afecta no dominar el idioma inglés, u otros aspectos que sí han sido discutidos, por ejemplo, la falta de investigación local (Buchanan, 2011). Generar conciencia acerca del impacto de las barreras idiomáticas en los Terapeutas Ocupacionales alrededor del mundo es relevante, considerando que, globalmente, en Terapia Ocupacional "el inglés continúa siendo el idioma de las ciencias" (Bannigan, 2011, p 5). Adicionalmente, los participantes demostraron tener poca información respecto a los recursos que sí están disponibles. Por ejemplo, sólo dos participantes identificaron como fuente de información la Revista Chilena de Terapia Ocupacional, y ninguno mencionó la versión en español del boletín de la Federación Mundial de Terapeutas Ocupacionales que es publicada esporádicamente.

Algunos participantes accedían a publicaciones científicas únicamente cuando éstas eran proveídas por docentes de los cursos que realizaban, hecho que podría significar un sesgo, pues dicha información ha sido preseleccionada por terceros. Se identificó el uso de blogs y páginas de Facebook como fuente de información disciplinar para guiar la práctica clínica. Aun cuando el uso de las redes sociales como estrategia para incrementar el conocimiento ha sido descrito en Terapia Ocupacional (Yan et al., 2012), resulta relevante para la disciplina en Chile poder evaluar cómo los recursos usados impactan la calidad de la práctica profesional. Resulta también interesante poder desarrollar estrategias para asegurar la calidad tanto de las traducciones de literatura, como de la información disponible en plataformas online, que fueron ampliamente utilizadas por los participantes de este estudio.

La incorporación de evidencia científica en la práctica es compleja y requiere adquisición de habilidades específicas (Metzler \& Metz, 2010). En este estudio sólo dos participantes señalaron haber recibido training en uso de bases de datos en la universidad, teniendo como requerimiento usar artículos científicos en exámenes finales. Las respuestas de los participantes en general sugieren que el currículo de los programas de Terapia Ocupacional no tiene integrado como concepto central la PBE, y que los esfuerzos aislados realizados, no logran real impacto en el trabajo diario de los Terapeutas. Este hallazgo es congruente con un artículo publicado en Chile por Aravena (2015), quien especuló que las destrezas y conocimientos requeridas para la PBE "podrían estar escasamente incorporados en los Terapeutas Ocupacionales y en su formación profesional" (p 190). Es trascendente que los profesionales que trabajan en academia en Chile puedan crear estrategias pedagógicas que introduzcan la PBE en las mallas curriculares, atravesando transversalmente los contenidos de todas las asignaturas y promoviendo el desarrollo de marcos de razonamiento clínico basado en evidencia.

La experticia clínica, una de las piedras angulares de la PBE, ha sido identificada como uno de los principales recursos de información usados por terapeutas ocupacionales para tomar decisiones clínicas (Copley \& Allen, 2009), consistente con los hallazgos de este estudio. Los participantes señalaron usar frecuentemente su intuición y opinión personal para tomar decisiones en la práctica, independiente de los años de experiencia profesional que posean, lo cual contrasta con la literatura que señala que con menos experiencia clínica, los terapeutas debieran guiarse en base a literatura y conocimiento disponible, y no en base a su intuición. Resultaría beneficioso para nuevas generaciones en Chile ser entrenados como "Terapeutas Ocupacionales críticos" capaces de identificar, usar y articular conocimiento de calidad para respaldar sus prácticas cotidianas (Robertson et al., 2015, p.68). Dicho entrenamiento elevaría el estatus de la profesión en el país, y mejoraría estándares de los servicios ofrecidos. La adopción de la PBE, y el desarrollo de un marco de razonamiento clínico basado en evidencia es una prioridad para Chile, en el contexto de ser un país en vías de desarrollo con el potencial de cuadriplicar el número actual de graduados en los próximos años.

El desarrollo de experticia clínica requiere perfeccionamiento continuo (Townsend et al., 2006), por tanto, resulta preocupante que la variedad de tópicos actualmente enseñados en Chile, esté limitado a un reducido número de modelos y teorías de intervención. Dichos modelos son ampliamente aplicados a todos los pacientes, y no siempre están en línea con las prácticas contemporáneas de Terapia Ocupacional en el mundo. Por ejemplo, la práctica centrada en la ocupación (Rodger, 2010) es reconocido a nivel mundial en pediatría, sin embargo, éste y otros modelos no fueron familiares para los terapeutas encuestados. El acceso limitado a la 
información indudablemente afecta la calidad de los servicios entregados a los niños en Chile. Asi, todos los participantes de este estudio que trabajaban con pacientes con parálisis cerebral señalaron usar IS y TND como estrategias de tratamiento, lo cual es corroborado por Navarrete (2013) quien señala que la TND es el tratamiento más común para los niños con parálisis cerebral en Chile. Paradójicamente, una revisión sistemática publicada por Novak y colaboradores (2013), sugiere que "ha sido demostrado que la TND e IS son inefectivas en niños con parálisis cerebral, y por lo tanto no se recomiendan" (p.90o) como estrategias de tratamiento. Resulta evidente la necesidad de crear planes de acción para que los Terapeutas en Chile accedan a conocimiento disciplinar actualizado, con el fin de entregar servicios eficientes a sus pacientes, e influir en que las políticas de capacitación de las instituciones en que trabajan vayan en la dirección correcta.

Se ha señalado que mecanismos de control dentro de la profesión, así como la presencia de mentores y líderes, son relevantes para el progreso de la misma (Lapointe et al., 2013). En este estudio, los participantes reportaron falta de mentores, y ausencia de supervisiones formales, situación diametralmente opuesta en países desarrollados, donde estas estrategias son parte de la cultura profesional y promovida por Asociaciones profesionales. Lograr avances en esta materia es importante, ya que aun cuando existe un código de ética profesional vigente en Chile, éste podría ser insuficiente para asegurar la calidad de la práctica y efectivamente proteger a sus usuarios.

En relación al rol de los niños en los procesos de intervención, este estudio evidencia que aun cuando los participantes tratan de incorporar las perspectivas y preferencias de los pacientes, estas acciones parecen estar más guiadas por su intuición que por un marco de referencia establecido, como es la práctica centrada en el cliente, un enfoque contemporáneo en salud que es relevante globalmente (Gupta \& Taff, 2015). Seis participantes señalaron consultar a los padres respecto a sus expectativas del tratamiento, sin embargo sólo uno de ellos, perteneciente a un centro privado, les permitía priorizar objetivos de tratamiento. Sólo un número reducido de participantes permitía a los padres participar activamente de las intervenciones. Investigaciones recientes en Terapia Ocupacional han demostrado que para determinados diagnósticos, las intervenciones enfocadas en la interacción de los niños con sus padres, resultan ser más efectivas que las intervenciones enfocadas exclusivamente en el niño (Barfoot et al., 2015).

En relación al contexto de práctica, este estudio mostró que factores como falta de tiempo y políticas institucionales impactan la toma de decisiones clínicas. Es interesante reflexionar en qué medida los terapeutas ocupacionales en Chile consideran estos factores, ya que en este estudio algunos participantes señalaron que carecen de los implementos mínimos para aplicar IS, sin embargo, es la alternativa de tratamiento elegida. Factores tales como escasas opciones de programas de postgrado, falta de conexión con la comunidad científica global y reducido acceso al conocimiento disciplinar emergieron como barreras para la PBE. Por lo tanto, resulta fundamental el instalar el tema en la agenda de discusión de la Terapia Ocupacional en Chile, y aunar esfuerzos de diversos actores para asegurar el progreso de la profesión en el futuro.

\section{LIMITACIONES}

Los participantes de este estudio fueron elegidos a conveniencia, asegurando máxima variación. Sin embargo los resultados pueden no ser representativos de la práctica de los terapeutas ocupacionales a lo largo de Chile, siendo necesario ser cauteloso al generalizar estos hallazgos. Dificultades en poder observar sesiones terapéuticas fue otra limitación, ya que podrían proveer de información adicional para triangular los datos. La traducción de los datos representó un riesgo al estudio, por tanto, éstas fueron revisadas por un traductor profesional y re-chequeadas por la investigadora principal. Finalmente, realizar el análisis de datos en Australia implicó riesgos de apreciar insuficientemente influencias culturales de Chile, siendo minimizados a través de un proceso de inmersión de la investigadora principal en la cultura profesional en Australia para informar las reflexiones.

\section{RECOMENDACIONES}

Se requieren futuras investigaciones en los diversos campos de acción de la Terapia Ocupacional en Chile, que permitan tener una mirada global y objetiva, e impulsar planes de desarrollo de la disciplina. Se considera importante que el Colegio de Terapeutas Ocupacionales se haga parte de estas acciones, que promuevan la PBE y la construcción de conocimiento local, también orientado a contribuir al desarrollo global de la profesión. 


\section{FINANCIAMIENTO}

La investigadora principal cuenta con financiamiento otorgado por Becas de Magíster en el extranjero de CONICYT, Gobierno de Chile. Esta institución sólo proporcionó soporte financiero a la investigadora principal, pero no tuvo ninguna injerencia en el diseño, recolección y análisis de datos, como tampoco con la difusión de sus resultados.

\section{AgradeCimientos}

Agradecemos a los diez Terapeutas Ocupacionales que participaron en este estudio y compartieron generosamente su tiempo y experiencia con el equipo investigador, haciendo posible la ejecución de este proyecto.

\section{RefERENCIAS BibliográfiCAS}

Aravena, J. (2015). ¿Quétanto entendemos el concepto de práctica basada en la evidencia en terapia ocupacional? Revista Chilena de Terapia Ocupacional, 15 (1), 189-192. doi:10.5354/0717-5346.2015.37142

Aravena, J., Gajardo, J., \& Molina, A. (2016). ¿Qué pensamos de la investigación en terapia ocupacional? Percepción de un grupo de estudiantes y docentes en Chile. Revista Chilena de Terapia Ocupacional, 16 (2) , 175-183. doi:10.5354/0717-5346.2016.44761

Alsop, A. (2013). Continuing professional development in health and social care: Strategies for lifelong learning/Auldeen Alsop. (2nd ed.). Chichester, West Sussex: Wiley-Blackwell.

Bannigan, K. (2011). Global approach to evidence based occupational therapy: what progress has been made since 2006? World Federation of Occupational Therapists Bulletin, 64(1), 4-6. doi:10.1179/ otb.2011.64.1.002

Barfoot, J., Meredith, P., Ziviani, J., \& Whittingham, K. (2015). Relationship-focused parenting intervention to support developmental outcomes for a young child with cerebral palsy: A practice application. British Journal of Occupational Therapy, 78(10), 640-643. doi: 10.1177/0308022615583304

Bazoret, E. (2006). El valor histórico del pituto: clase media, integración y diferenciación social en Chile. Revista de Sociología, 20, 69-96. Retrieved from hhhp://www.facso.uchile.cl/publicaciones/ sociologia/articulos/20/2003-Bazoret.pdf

Bennett, S., \& Bennett, J. (2000). The process of evidence-based practice in occupational therapy: informing clinical decisions. Australian Occupational Therapy Journal, 47(4), 171.

Bennett, S., Tooth, L., McKenna, K., Rodger, S., Strong, J., Ziviani, J. Gibson, L. (2003). Perceptions of evidence-based practice: A survey of
Australian occupational therapists. Australian Occupational Therapy Journal, 50(1), 13-22. doi: 10.1046/j.1440-1630.2003.00341.x

Buchanan, H. (2011). The uptake of evidence-based practice by occupational therapists in South Africa. World Federation of Occupational Therapists Bulletin, 64(1), 29-38. doi:10.1179/otb.2011.64.1.00

Colquhoun, H., Letts, L., Law, M., MacDermid, J., \& Missiuna, C. (2010). A scoping review of the use of theory in studies of knowledge translation. Canadian Journal of Occupational Therapy, 77(5), 270-279.

Copley, J., Nelson, A., Turpin, M., Underwood, K., \& Flanigan, K. (2008). Factors influencing therapists' interventions for children with learning difficulties. Canadian Journal of Occupational Therapy, 75(2), 105-113.

Copley, J., \& Allen, S. (2009). Using all the available evidence: perceptions of paediatric occupational therapists about how to increase evidence-based practice. International Journal of Evidence-Based Healthcare, 7(3), 193-200. doi: 10.1111/j.1744-1609.2009.00137.x

Copley, J., Bennett, S., \& Turpin, M. (2010a). Decision Making for Occupation-Centred Practice with Children. Occupation-Centred Practice with Children (pp. 320-341): Wiley-Blackwell.

Copley, J., Turpin, M., \& King, T. (2010b). Information used by an expert paediatric occupational therapist when making clinical decisions. Canadian Journal of Occupational Therapy, 77(4), 249-256.

Dysart, A., \& Tomlin, G. (2002). Factors related to evidence-based practice among U.S. occupational therapy clinicians. The American Journal of Occupational Therapy: Official Publication of the American Occupational Therapy Association, 56(3), 275-284. doi:10.5014/ajot.56.3.275

García, J., Copley, J., Turpin, M., Chien, C.W., \& Peña, N. (2016). Continuing professional development in Chile: A case study. World Federation of Occupational Therapists Bulletin, 1-4. doi: 10.1080/14473828.2016.1207898

Gómez, S. (2012). Antecedentes, creación y desarrollo de la Terapia ocupacional en Chile. Santiago, Chile.

Gupta, J., \& Taff, S. (2015). The illusion of client-centred practice. Scandinavian Journal of Occupational Therapy, 2015, Vol. 22(4), P.244-251, 22(4), 244-251.

Gustafsson, L., Molineux, M., \& Bennett, S. (2014). Contemporary occupational therapy practice: the challenges of being evidence based and philosophically congruent. Australian Occupational Therapy Journal, 67(2), 121-123. doi: 10.1111/1440-1630.12110

Hoffmann, T., Bennett, S., \& Del Mar, C. (2013). Evidence-based practice across the health professions/Tammy Hoffmann, Sally Bennett, Chris DelMar (2nd ed.). Chatswood, N.S.W.: Chatswood, N.S.W.: Elsevier Australia.

Hu, D., Burke, J., Aliki, T. (2012) Occupational therapists' involvement views, and training needs of evidence-based practice: a rural perspective. International Journal of Therapy and Rehabilitation, 19(11), 618-628. 
Humphris, D., Littlejohns, P., Victor, C., O'Halloran, P., \& Peacock, J. (2000). Implementing Evidence-Based Practice: Factors that Influence the Use of Research Evidence by Occupational Therapists. The British Journal of Occupational Therapy, 63(11), 516-522. doi: $10.1177 / 030802260006301102$

Ilott, I. C., Taylor, M., \& Bolaños, C. (2006). Evidence-based occupational therapy: It's time to take a global approach. British Journal of Occupational Therapy, 69(1), 38-41.

Lapointe, J., Baptiste, S., Von Zweck, C., \& Craik, J. (2013). Developing the occupational therapy profession through leadership and mentorship: Energizing opportunities. World Federation of Occupational Therapists Bulletin, 68(1), 38.

Lin, S., Murphy, S., \& Robinson, J. (2010). Facilitating evidence-based practice: process, strategies, and resources. American Journal of Occupational Therapy, 64(1), 164-171.

Metzler, M., \& Metz, G. (2010). Translating knowledge to practice: an occupational therapy perspective. Australian Occupational Therapy Journal, 57(6), 373-379. doi: 10.1111/j.1440-1630.2010.00873.x

Mroz, T., Pitonyak, S., Fogelberg, D., \& Leland, N. (2015). Client centeredness and health reform: Key issues for occupational therapy. AJOT: American Journal of Occupational Therapy, 69(5)

Navarrete, M. (2013) Terapia Ocupacional y su relación con la infancia. ContexTO, ISSN 0719-1707, Año 2, 2013. №2, pp. 11-23. Retrieved from http://www.ucentral.cl/prontus_ucentral2012/site/ artic/20140812/asocfile/20140812173222/contexto_2014.pdf

Novak, I., McIntyre, S., Morgan, C., Campbell, L., Dark, L., Morton, N., Goldsmith, S. (2013). A systematic review of interventions for children with cerebral palsy: state of the evidence. Developmental Medicine and Child Neurology, 55(10), 885-910. doi: 10.1111/dmcn.12246.

Piergrossi, J. (2004). A Question of Priorities: Notes from The International Conference on Evidence-Based Occupational Therapy July 11-14, 2004, Washington D.C., U.s.A. World Federation of Occupational Therapists Bulletin, 50(1), 56.

Robertson, D., Warrender, F., \& Barnard, S. (2015). The critical occupational therapy practitioner: How to define expertise? Australian Occupational Therapy Journal, 62(1), 68-71.

Rodger, S. (2010). Occupation-centred practice with children: A practical guide for occupational therapists / Sylvia Rodger. Chichester, U.K.; Malden, MA: Wiley/Blackwell Pub.

Rycroft-Malone, J., Seers, K., Titchen, A., Harvey, G., Kitson, A., \& McCormack, B. (2004). What counts as evidence in evidence-based practice? Journal of Advanced Nursing, 47(1), 81-90. doi: 10.1111/j.1365-2648.2004.03068.x

Sackett, D., Rosenberg, W., Gray, J., Haynes, R., \& Richardson, W. (1996). Evidence based medicine: what it is and what it isn't. British Medical Journal, 312(7023), 71-72.
Shafaroodi, N., Kamali, M., Parvizy, S., Mehraban, A. H., \& O’Toole, G. (2014). Factors affecting clinical reasoning of occupational therapists: a qualitative study. Medical Journal Islamic Republic of Iran, $28,8$.

Thomas, A., \& Law, M. (2013). Research utilization and evidence-based practice in occupational therapy: a scoping study. American Journal of Occupational Therapy, 67(4), e55-65. doi: 10.5014/ ajot.2013.00639

Thomas, A., Saroyan, A., \& Lajoie, S. (2012). Creation of an evidence-based practice reference model in falls prevention: findings from occupational therapy. Disability and Rehabilitation, 34(4), 311-328. doi: 10.3109/09638288.2011.60721

Thorne, S. (2008). Interpretive description (Vol. 2.). Walnut Creek, CA: Left Coast Press.

Tse, S., Lloyd, C., Penman, M., King, R., \& Bassett, H. (2004). Evidence-based practice and rehabilitation: Occupational therapy in Australia and New Zealand experiences. International Journal of Rehabilitation Research, 27(4), 269-274.

Townsend, E. L., Sheffield, S., Stadnyk, R., \& Beagan, B. (2006). Effects of workplace policy on continuing professional development: The case of occupational therapy in Nova Scotia, Canada. Canadian Journal of Occupational Therapy, 73(2), 98-108.

Yan, Y., Sinclair, K., \& Penman, M. (2012). Using social networking for professional_development of occupational therapy throughout the people's Republic of China. World Federation of Occupational Therapists Bulletin, 66, 47. 